\title{
Small bowel perforation with multiple intestinal metastases from lung carcinoma: A case report
}

\author{
EVANGELOS P. MISIAKOS ${ }^{1}$, ALINA-ROXANI GOULOUMI ${ }^{2}$, DIMITRIOS SCHIZAS ${ }^{3}$, VASILEIA DAMASKOU ${ }^{2}$, \\ DIMITRIOS TSAPRALIS ${ }^{4}$, FREDERICK-ANTHONY FARRUGIA ${ }^{1}$, NIKOLAOS MACHAIRAS $^{1}$, \\ DIMITRIOS PAPACONSTANTINOU $^{1}$, ARIADNI TZANETI ${ }^{1}$ and ANASTASIOS MACHAIRAS ${ }^{1}$
}

\author{
${ }^{1}$ 3rd Department of Surgery; ${ }^{2}$ 2nd Department of Pathology, National and Kapodistrian University of Athens, \\ School of Medicine, Attikon Hospital, Athens $12462 ;{ }^{3} 1$ st Department of Surgery, National and Kapodistrian \\ University of Athens, School of Medicine, Laiko Hospital, Athens $11527 ;{ }^{4}$ Department of General Surgery, \\ General Hospital/Health Center of Ierapetra, Crete 77200, Greece
}

Received June 11, 2018; Accepted January 23, 2019

DOI: $10.3892 / 01.2019 .10031$

\begin{abstract}
The present report describes the case of an 81-year-old woman who underwent an emergency explorative laparotomy due to small bowel perforation. Diffuse feculent peritonitis due to perforation of the jejunum was identified. In addition, six distinct tumors of the jejunum in close proximity to the perforation site, as well as two more lesions caudally at the ileum, were identified. A separate stenotic tumor was identified at the sigmoid colon, causing moderate dilatation of the whole intestine. An enterectomy, including the perforation site and the proximal six tumors, was performed, followed by limited enterectomies for the distal two tumors and side-to-side anastomoses of the small intestine. All resected tumors were identified at histology as metastatic from lung carcinoma. Postoperatively, the patient remained in a severe septic condition and succumbed the following day. Metastatic lesions from lung carcinoma in the small bowel wall are a rare finding predisposing to bowel perforation. When an acute condition coexists with multiple intestinal metastases, the mortality rate is high despite prompt surgical intervention.
\end{abstract}

\section{Introduction}

Lung cancer typically has a poor prognosis, as metastases are often present at the time of diagnosis (1). Distal metastases are more commonly found in the adrenal glands, liver, bone, brain and kidney; metastases located along the gastrointestinal tract are very rare and account for a small number of patients $(2,3)$.

Correspondence to: Dr Evangelos P. Misiakos, 3rd Department of Surgery, National and Kapodistrian University of Athens, School of Medicine, Attikon Hospital, 1 Rimini, Chaidari, Athens 12462, Greece

E-mail: misiakos@med.uoa.gr

Key words: lung, carcinoma, small bowel, gastrointestinal neoplasms, metastasis, perforation
More specifically, such metastatic lesions are more commonly encountered in the advanced stages of the disease and are consequently associated with unfavorable disease prognosis. With regards to associated symptomatology, when the metastatic disease involves the upper gastrointestinal tract, bleeding is the most common symptom, whilst when the small intestine is involved, the most typical manifestation is intestinal obstruction, with or without perforation (4). Herein, a rare case of a surgical patient with acute abdomen due to small bowel perforation and multiple intestinal metastases from lung carcinoma is reported.

\section{Case report}

An 81-year-old woman was admitted to the emergency department of our institution due to fatigue and malaise. Upon admission the patient was hemodynamically stable. The patient's history of atrial fibrillation was significant and a Coumadin analog was administered. Additionally, the patient had a history of a locally advanced non-small cell carcinoma of the right lung (stage IIIB); her lesion was considered unresectable at the time and the patient received cisplatin and etoposide combined with radiotherapy, showing a significant response. Approximately 1.5 years later, she was diagnosed with a metastatic lesion of the left lung during follow-up. Chemotherapy was restarted, but the patient was lost to follow-up in the next year. The patient reported mild abdominal discomfort, which had started 2 weeks prior to the current presentation. Upon admission, she presented with severe anemia (Hct 18.5\%), leucopenia (WBC 2,330 K/ $/ \mu 1$ ), thrombocytopenia (PLT 55,000 K/ $\mu \mathrm{l}$ ), and a protracted INR of 6.41. Chest X-ray showed diffuse pulmonary infiltrations and lobular pneumonia at the right lower lobe. The abdominal ultrasound showed multiple focal lesions in both hepatic lobes, with features of metastasis. At 2 days post-admission, the patient presented with diffuse abdominal pain combined with fever $\left(38.1^{\circ} \mathrm{C}\right)$, and surgical consultation was demanded. At physical examination the patient had diffuse abdominal tenderness and guarding, rebound tenderness, abdominal distention and obstipation. Her blood test revealed Hct 22.1\%, 
WBC $20,000 \mathrm{~K} / \mu 1$, PLT $47,000 \mathrm{~K} / \mu 1$, whereas the biochemical tests were within normal limits. An abdominal CT revealed wall thickening at the level of the mid-sigmoid and the presence of free air in the peritoneal cavity, likely due to perforation of the gastrointestinal tract. Several round lesions were infiltrating the wall of the small bowel, $2-3 \mathrm{~cm}$ in diameter, and large metastatic lesions were also revealed within the liver parenchyma. A moderate quantity of ascitic fluid, and multiple enlarged lymph nodes within the mesentery were also identified (Fig. 1). The patient was urgently led to surgery for an exploratory laparotomy. Upon entering the peritoneal cavity, diffuse feculent peritonitis, due to perforation of the jejunum $1 \mathrm{~m}$ from the ligament of Treitz, was found. In addition, six distinct tumors of the jejunum close to the perforation site were found, as well as two more similar lesions caudally at the ileum (Fig. 2A). The tumors were round, hard and protruding from the bowel wall, with dimensions $<4 \mathrm{~cm}$. A separate stenotic tumor at the wall of the mid-sigmoid was identified, which caused moderate dilation of the proximal intestine (Fig. 2B). Enterectomy, including the perforation site and the proximal six tumors, was first performed, followed by limited enterectomies for the distal two tumors (Fig. 2C and D). The continuity of the gastrointestinal tract was reinstated with side-to-side anastomoses of the small intestine using a linear stapler. For the sigmoid tumor, a Hartmann procedure with limited resection of the mid-sigmoid was performed. The patient remained in a severe septic condition following surgery and succumbed the next day.

The resected specimens consisted of three segments of small bowel and a segment of large bowel measuring 85, 6.4, 4.7 and $9.2 \mathrm{~cm}$ in length, respectively. Macroscopic examination of the resected bowel revealed six tumors with a maximum diameter from 2.4 to $4.9 \mathrm{~cm}$; the jejunal segment exhibited a point of serosal involvement and perforation. Additional findings included two tumors measuring 3.7 and $3.5 \mathrm{~cm}$, found in the other two segments of the small bowel. Upon examination of the large bowel, an intraluminal polypoid tumor measuring $1.1 \mathrm{~cm}$ with a mass of $3.3 \mathrm{~cm}$ in the pericolic fat was noted. On microscopic examination, hematoxylin and eosin (H\&E)-stained sections from the tumors showed features of a poorly differentiated neoplasm (Fig. 3) in the submucosa. By contrast, the intraluminal large bowel tumor was an adenoma with low-grade dysplasia. The possibility of numerous metastases was the primary consideration. The differential diagnosis was that of multiple intestinal neoplasms, either in the setting of familial adenomatous polyposis or associated with Lynch syndrome. Molecular analysis revealed mutation of p.Gly12Val (c.35 G>T) in KRAS proto-oncogene, GTPase (KRAS), with no mutations in NRAS proto-oncogene, GTPase (NRAS) or B-Raf proto-oncogene, serine/threonine kinase (BRAF). Immunohistochemical (IHC) testing for Mismatch Repair (MMR) proteins showed intact nuclear expression. Further IHC analysis performed on sections of a small bowel mass showed strong immunoreactivity for cytokeratin (CK)7, CK 8/18 and, particularly, thyroid transcription factor 1 (TTF1; Fig. 4A and B). Analysis of the pericolic fat mass additionally showed focal immunoreactivity for polyclonal carcinoembryonic antigen (pCEA), p63 (Fig. 4C) and CK5/6. Both tumors were negative for chromogranin and synaptophysin. Histochemical analysis with Alcian blue did not

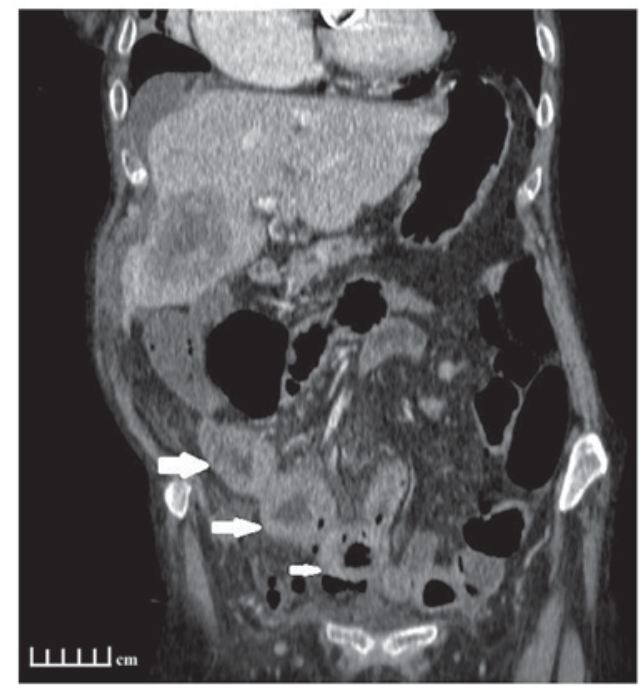

Figure 1. Abdominal computed tomography (coronal view) demonstrating a large metastatic tumor occupying a significant part of the liver parenchyma, and multiple tumors in a number of loops of the small intestine (arrows) infiltrating the bowel wall.

detect mucin. Based on this profile, a diagnosis of metastatic lung carcinoma-likely adenocarcinoma or adenosquamous carcinoma-was rendered. Finally, Napsin A staining was performed, which was also positive (Fig. 4D).

\section{Discussion}

Metastases to the gastrointestinal tract are rather rare in patients with lung cancer and are more commonly encountered in the advanced stages of the disease. The spread to the gastrointestinal tract occurs via the hematogenous and lymphatic routes. When the metastatic disease involves the upper gastrointestinal tract, the most common symptom is bleeding, but when it involves the small intestine, the most typical manifestation is intestinal obstruction, with or without perforation (4). Historically, Oschner and Debakey in 1942 reported gastrointestinal involvement in 4.3\% of 3,047 autopsies (5). Further, Yang et al (6) estimated the incidence of symptomatic gastrointestinal secondaries from primary lung cancer at $1.77 \%$. Other reports have shown that the prevalence of these tumors at autopsy is much higher, ranging from 4.7 to $14 \%(7,8)$. Such higher rates are attributed to the fact that most patients with small bowel metastases often present with non-specific symptoms. McNeill et al (2) reviewed 431 patients with diagnosed lung cancer and demonstrated that small bowel metastases were present in $10.7 \%$ of these patients at autopsy. All patients with small bowel metastases had at least one more metastatic site, with an average of 4.8 sites (2). In a published series of 423 autopsies, in which 58 patients presented with gastrointestinal tract secondary lesions from primary lung cancer, the most frequently encountered histological types were squamous cell (33\%), large cell (29\%) and oat cell $(19 \%)(7,9)$.

Perforation of the small intestine is an extremely rare and serious complication in lung cancer patients, occurring at the advanced stages of the disease. Morgan et al (10) reported the first case in 1961, in a patient with advanced lung carcinoma 

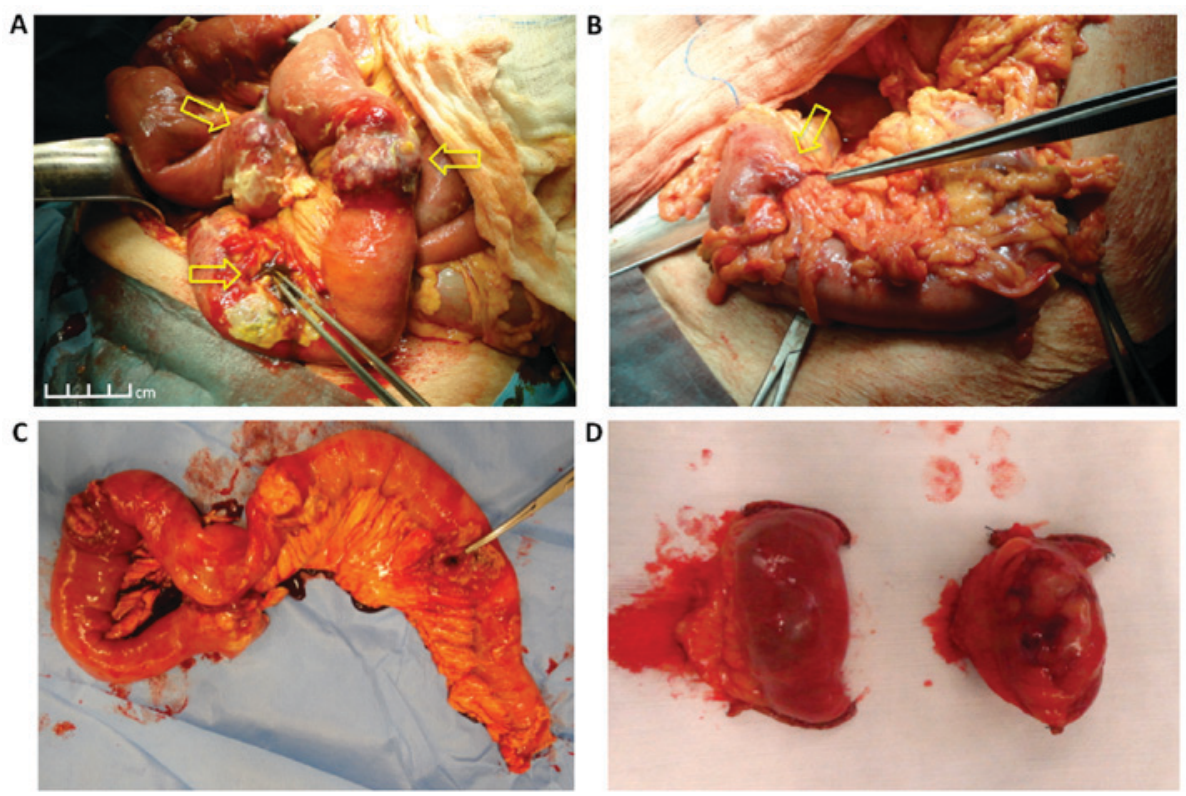

Figure 2. Intraoperative results. (A) Perforation site in the mid-jejunum and multiple tumors (arrows) of the jejunal wall close to the perforation site. (B) Stenotic tumor at the wall of the mid-sigmoid (arrow). (C) Enterectomy including the perforation site and the proximal six tumors of the small intestine. (D) Segmental enterectomies for the two tumors at the ileum.
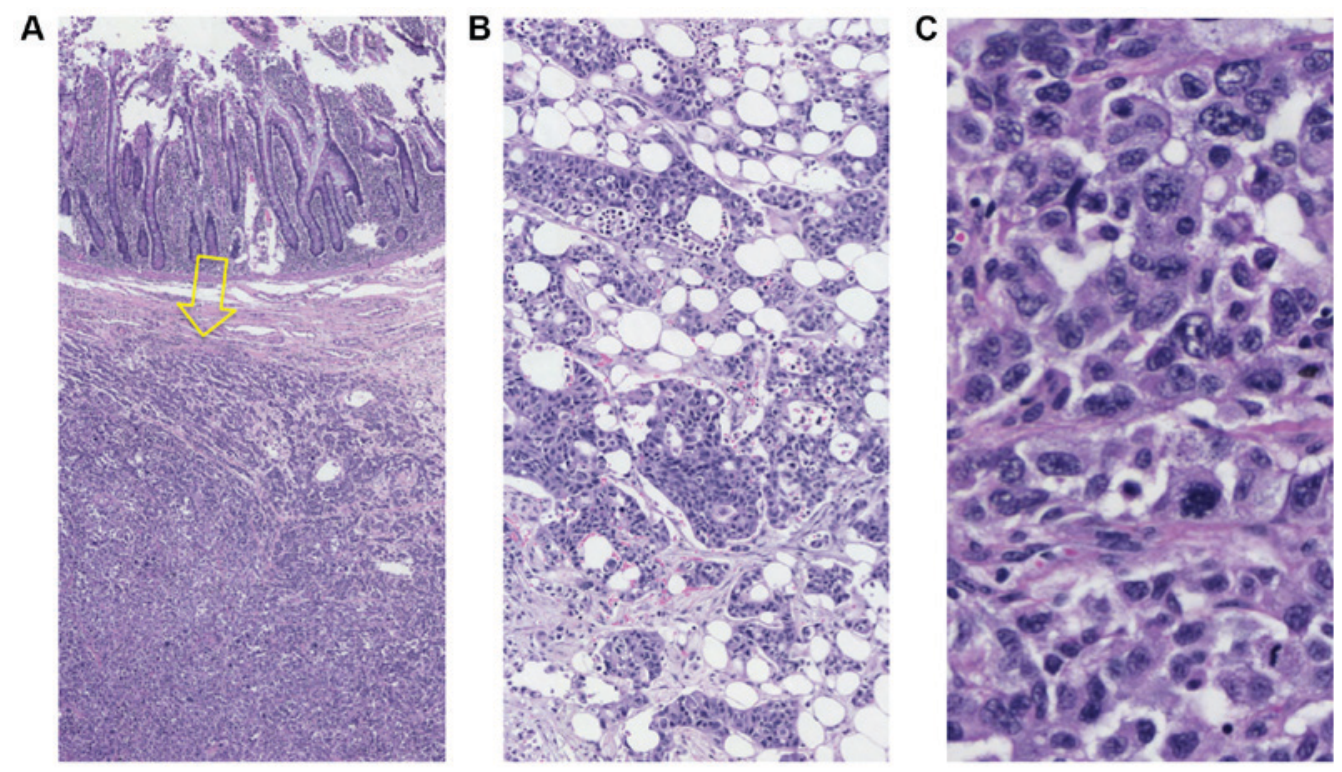

Figure 3. Histopathology of the resected lesions. (A) Sections demonstrated a poorly differentiated neoplasm in the small bowel submucosa (arrow) not associated with mucosal dysplasia (H\&E; magnification, $\mathrm{x} 4)$, and (B) in the pericolic fat (H\&E; magnification, x10). (C) Tumor cells at high magnification (H\&E; magnification, $\mathrm{x} 40)$. H\&E, hematoxylin and eosin.

receiving cyclophosphamide. Garwood et al (11) performed a Medline search to identify all cases of gastrointestinal tract perforations attributed to metastatic lung cancer reported in the literature. Data were collected from the medical literature between 1960 and 2005. They identified 98 cases of perforated lung cancer metastasis to the small intestine. Perforations occurred most often in the jejunum (53\%) followed by the ileum (28\%), whereas combined jejunum-ileum lesions accounted for $4 \%$ of perforations. Other causes of small bowel perforation included adenocarcinoma $(23.7 \%)$, squamous cell carcinoma (22.7\%), large cell carcinoma (20.6\%), and small cell carcinoma (19.6\%) (11).
The tumor replaces all or part of the bowel wall, resulting in various presentations: Bulky tumors causing lumen obstruction, necrotic tumors that often perforate, ulcerative lesions causing bleeding, or extensive mucosal surface involvement leading to malabsorption (12). McNeill et al (2) showed that when the primary cancer is in the lungs, metastases at the small bowel are more likely to perforate, as the metastatic tumors typically undergo necrosis prior to becoming large enough to cause intestinal obstruction (2).

At endoscopy, lung cancer metastases to the intestinal wall have no specific features, and usually appear as diffuse involvement of the intestinal mucosa, multiple nodules with/without 

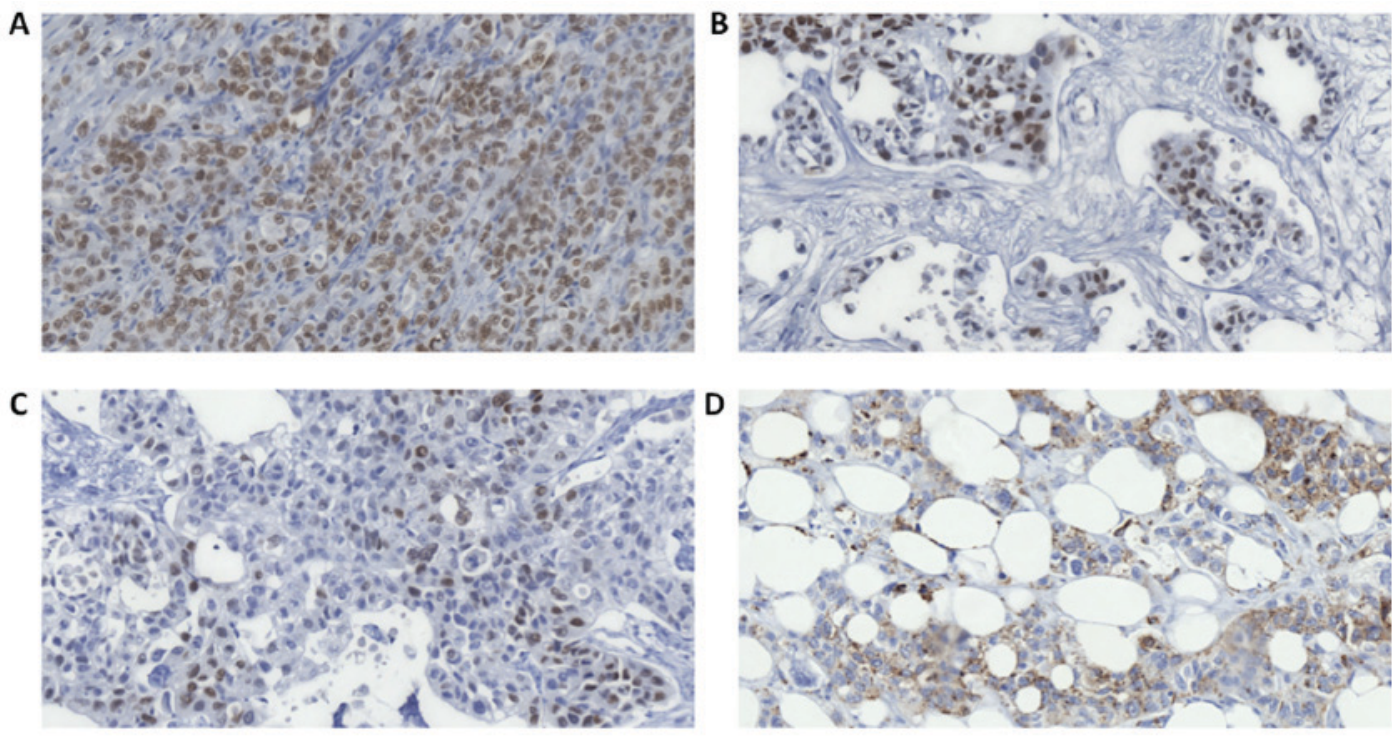

Figure 4. Immunohistochemical analysis of the resected specimens. (A) TTF1 positive staining (magnification, x20) in sections from a small bowel mass, (B) pericolic fat, (C) p63 and (D) Napsin A staining was additionally positive. TTF1, thyroid transcription factor 1.

mucosal ulceration, or as a solitary 'volcano-like' tumor of the intestine $(4,13)$. There has been a debate regarding the most common lung cancer histotype that metastasizes in the gastrointestinal tract. Some authors have suggested squamous cell carcinoma as the most common histological type $(4,14)$, whereas other reports have revealed that poorly differentiated pulmonary adenocarcinomas and large cell undifferentiated carcinomas have a specific predilection for the digestive tract $(4,7,15)$. Studies from Italy (4) and Taiwan (6) have suggested that $0.5-1.7 \%$ of patients with primary lung cancer develop gastrointestinal tract metastasis. The cell type in Taiwan was squamous cell carcinoma (3/6) in the majority of cases, whereas large cell carcinoma (10/18) was the dominant type in Italy. Therefore, the histological type predominantly associated with gastrointestinal metastasis remains unclear.

Gray et al (16) reported a case of small bowel perforation in a patient with non-small cell lung cancer following treatment with bevacizumab (16). Although several reports followed, the pathogenesis of perforation is not yet clear. Leidich and Rudolf (17) reported that perforations occur once the bowel wall is replaced by tumor cells, which is followed by cellular necrosis (17). However, chemotherapy also plays an important role in the development of bowel perforation. In this regard, Owen and Chasen (18) reported a case of intestinal perforation in a patient with an extrapulmonary large cell carcinoma of the small bowel, who received platinum-based chemotherapy. Chemotherapy may have induced rapid tumor necrosis, resulting in the loss of bowel wall integrity (18). Patients with small bowel perforation due to metastatic lung cancer typically present with signs of acute abdomen. Management consists of patient resuscitation, stabilization with fluids and electrolytes, followed by exploratory laparotomy. At surgery, resection of the affected part of the bowel, including the site of perforation, should be performed, with or without prophylactic stoma formation (17,19). Meticulous exploration and irrigation of the peritoneal cavity should also be performed on an emergency basis and is the only way to revert ongoing sepsis. Despite the poor prognosis of these patients, surgery may provide acceptable palliation and lead to satisfactory survival $(19,20)$. Indeed, Nagashima et al (21) reviewed 48 cases operated on for intestinal perforation due to metastatic lung cancer, and reported a median postoperative survival time of 48 days. They also concluded that patient performance status may be an important prognostic factor (21).

Pathological diagnosis with the use of immunohistochemical stains is the only reliable way to differentiate a primary small bowel malignancy from a metastatic lesion deriving from the lung (22). Primary lung carcinomas usually exhibit a $\mathrm{CK} 7^{+} / \mathrm{CK} 20^{-}$immunophenotype as opposed to the usual $\mathrm{CK} 77^{-} / \mathrm{CK} 20^{+}$pattern of intestinal adenocarcinomas (4). However, primary rectal or small bowel adenocarcinomas may also be $\mathrm{CK}^{+} / \mathrm{CK} 20^{-}$(8). Thus, TTF-1 and or Napsin A positivity is critical in establishing a primary lung cancer origin. TTF-1 is highly specific for adenocarcinomas of pulmonary origin exhibiting a positive predictive value of $>90 \%(4,19)$.

Finally, according to the most recent review of the literature, surgical resection of the involved portion of the small bowel with primary end-to-end anastomosis may be the optimal treatment strategy, particularly in cases with obstruction, hemorrhage and/or perforation (23). Notably, following successful resection of such lesions and despite the presence of additional metastatic lesions besides the intestinal tract, survival for $>1$ year has been reported. However, in the vast majority of these patients, survival outcomes are poor (24).

In conclusion, metastatic lesions from lung carcinoma in the small bowel wall are a rare finding predisposing to bowel perforation. Perforation peritonitis associated with multiple intestinal metastases carries a high mortality rate, even with prompt surgical therapy.

\section{Acknowledgements}

Not applicable. 


\section{Funding}

No funding was received.

\section{Availability of data and materials}

All data generated or analyzed during the current study are included in this published article.

\section{Authors' contributions}

EPM and AM contributed to the conception and design of the study. ARG, VD, DT, DP, FAF, AT, DS and NM contributed to the acquisition, analysis and interpretation of data. EPM, DS and NM drafted the manuscript and/or revised it critically for important intellectual content. All authors read and approved the final manuscript.

\section{Ethics approval and consent to participate}

Not applicable.

\section{Patient consent for publication}

Written informed consent was obtained from the sister of the patient, who was the next of kin for this patient, for publication of this case report and any accompanying images.

\section{Competing interests}

The authors declare that they have no competing interests.

\section{References}

1. Liu W, Zhou W, QL WL, Ma YD and Xu YY: Gastrointestinal hemorrhage due to ileal metastasis from primary lung cancer. World J Gastroenterol 21: 3435-3440, 2015.

2. McNeill PM, Wagman LD and Neifeld JP: Small bowel metastases from primary carcinoma of the lung. Cancer 59: 1486-1489, 1987.

3. Hoffman PC, Mauer AM and Vokes EE: Lung cancer. Lancet 355: 479-485, 2000.

4. Rossi G, Marchioni A, Romagnani E, Bertolini F, Longo L, Cavazza A and Barbieri F: Primary lung cancer presenting with gastrointestinal tract involvement: Clinicopathologic and immunohistochemical features in a series of 18 consecutive cases. J Thorac Oncol 2: 115-120, 2007.

5. Oschner A and Debakey M: Significance of metastasis in primary carcinoma of the lungs: report of two cases with unusual sites of metastasis. J Thorac Surg 11: 357-387, 1942.

6. Yang CJ, Hwang JJ, Kang WY, Chong LW, Wang TH, Sheu CC, Tsai JR and Huang MS: Gastro-intestinal metastases of primary lung carcinoma: Clinical presentations and outcome. Lung Cancer 54: 319-323, 2006.
7. Antler AS, Ough Y, Pitchumoni CS, Davidian M and Thelmo W: Gastrointestinal metastases from malignant tumors of the lung. Cancer 49: 170-172, 1982.

8. Yoshimoto A, Kasahara E and Kawashima A: gastrointestinal metastases from primary lung cancer. Eur J Cancer 42: 3157-3160, 2006.

9. Yuen JS, Chow PK and Ahmed Q: Metastatic lung cancer causing bowel perforations: Spontaneous or chemotherapy-related? ANZ J Surg 72: 245-246, 2002.

10. Morgan MW, Sigel B and Wolcott MW: Perforation of a metastatic carcinoma of the jejunum after cancer chemotherapy. Surgery 49: 687-689, 1961.

11. Garwood RA, Sawyer MD, Ledesma EJ, Foley E and Claridge JA: A case and review of bowel perforation secondary to metastatic lung cancer. Am Surg 71: 110-116, 2005.

12. Sujith NS, Sima RR, Thimmappa A and Govindaraj S: An unusual presentation of metastatic lung cancer. Int Surg J 3: $1700-1704,2016$

13. Hsu CC, Chen JJ and Changchien CS: Endoscopic features of metastatic tumors in the upper gastrointestinal tract. Endoscopy 28: 249-253, 1996.

14. Berger A, Cellier C, Daniel C, Kron C, Riquet M, Barbier JP, Cugnenc $\mathrm{PH}$ and Landi B: Small bowel metastases from primary carcinoma of the lung: Clinical findings and outcome. Am J Gastroenterol 94: 1884-1887, 1999.

15. Edwards R and Royle G: Metastatic carcinoma causing haematemesis. Br Med J 2: 598, 1975.

16. Gray J, Murren J, Sharma A, Kelley S, Detterbeck F and Bepler G: Perforated viscus in a patient with non-small cell lung cancer receiving bevacizumab. J Thorac Oncol 2: 571-573, 2007.

17. Leidich RB and Rudolf LE: Small bowel perforation secondary to metastatic lung carcinoma. Ann Surg 193: 67-69, 1981.

18. Owen S and Chasen M: Chemotherapy-induced small bowel perforation in a patient with extrapulmonary small-cell carcinoma of the small bowel. Curr Oncol 15: 298-301, 2008.

19. Goh BK, Yeo AW, Koong HN, Ooi LL and Wong WK: Laparotomy for acute complications of gastrointestinal metastases from lung cancer: Is it a worthwhile or futile effort? Surg Today 37: 370-114, 2007.

20. Salemis NS, Nikou E, Liatsos C, Gakis C, Karagkiouzis G and Gourgiotis S: Small bowel perforation secondary to metastatic non-small cell lung cancer. A rare entity with a dismal prognosis. J Gastrointest Cancer 43: 391-395, 2012.

21. Nagashima Y, Okamoto H, Narita Y, Hida N, Naoki K, Kunikane $\mathrm{H}$ and Watanabe $\mathrm{K}$ : Perforation of the small intestine caused by metastasis from primary lung cancer: Report of two cases and the discussion of 48 cases published in the Japanese literature. Nihon Kokyuki Gakkai Zasshi 45: 430-435, 2007 (In Japanese)

22. Gonzalez-Tallon AI, Vasquez-Guerrero J and Garcia-Mayor MA: Colonic metastases from lung carcinoma: A case report and review of the literature. Gastroenterology Res 6: 29-33, 2013.

23. Di JZ, Peng J and Wang ZG: Prevalence, clinicopathological characteristics, treatment, and prognosis of intestinal metastasis of primary lung cancer: A comprehensive review. Surg Oncol 23: 72-80, 2014.

24. Yildirim M, Tasli F, Bayam ME and Postaci H: A rare cause of small bowel transection: Metastatic lung cancer. Med Princ Pract 19: 232-234, 2010.

This work is licensed under a Creative Commons Attribution-NonCommercial-NoDerivatives 4.0 International (CC BY-NC-ND 4.0) License. 